\title{
Surface structuring of zirconium-based bulk metallic glasses using ultrashort laser pulses
}

\author{
Claus Dold ${ }^{a}$, Martin Kachel ${ }^{b}$, Dirk Wortmann ${ }^{b}$, Victor Wessels ${ }^{c}$, Andreas Dohrn ${ }^{d}$, Arie \\ Bruinink $^{e}$, Frank Pude ${ }^{a}$, Joerg F. Loeffler ${ }^{c}$, Reinhart Poprawe ${ }^{b, d}$, Konrad Wegener ${ }^{a, f}$ \\ ${ }^{a}$ Inspire AG, Tannenstrasse 3, Zurich, Switzerland; \\ ${ }^{b}$ Chair of laser technology (LLT), RWTH, Aachen, Germany; \\ ${ }^{c}$ Laboratory of metal physics and technology (LMPT), ETH, Zurich, Switzerland \\ ${ }^{d}$ Institute for laser technology (ILT), Aachen, Germany \\ ${ }^{e}$ Laboratory of materials-biology interactions, EMPA, St. Gallen, Switzerland

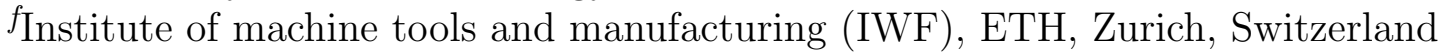

\begin{abstract}
A technique for structuring the surface of a bulk metallic glass (BMG) via scanning with a beam of laser pulses in the pico- and femtosecond time regime is presented. Specimens were characterized by various techniques to analyze the effects of ultrashort laser pulses on the amorphous matrix. Broadly varying surface structures, with roughness parameters in the range of $R_{\mathrm{a}}=0.066$ to $0.329 \mu \mathrm{m}$, measured using white light interferometry (WIM) and optical 3D microscopy, were produced. These techniques could be useful for fabricating biomedical implants from BMGs. As proof of principle, a patterned grid, designed for evaluating bone cell response to different surface structures, are produced.
\end{abstract}

Keywords: Ultrafast laser processing, bulk metallic glass, surface structuring

\section{INTRODUCTION}

A metallic glass is an alloy that possesses an atomic structure without long-range order, similar to that of a liquid metal. ${ }^{1}$ This disordered, or amorphous, structure gives rise to material properties that are often superior to traditional crystalline alloys. Owing to its lack of a crystalline lattice, and the associated grain boundaries and defects, metallic glass typically has higher strength (nearly $4 \mathrm{GPa}$ for some alloys), hardness and corrosion resistance. ${ }^{2,3}$ Amorphous alloys also possess a higher elastic limit, typically 2 to $5 \%$, which makes them intriguing for mechanical energy storage applications, such as springs. ${ }^{4}$ The unique thermoplastic forming (TPF) ability of metallic glasses ${ }^{5,6}$ has been exploited to produce complex parts, including for micro- and nano-scale devices, using techniques borrowed from polymer technology. ${ }^{7,8}$ The cooling rates necessary to "freeze-in" a liquid-like atomic arrangement from the melt are often rapid, sometimes on the order of $10^{5} \mathrm{~K} / \mathrm{s}^{1}$ Early work, such as the original discovery of metallic glass in a $\mathrm{Au}-\mathrm{Si}$ alloy, ${ }^{9}$ used techniques like roller melt-spinning and splat quenching to achieve rapid cooling, but such methods limit the dimension of the work-piece to ribbons or foils of about 10 to $50 \mu \mathrm{m}$ in thickness. More recently, amorphous alloys possessing much slower critical cooling rates, on the order of 100 to $102 \mathrm{~K} / \mathrm{s}$ were discovered, allowing for fabrication of much larger amorphous specimens with critical dimensions of up to $72 \mathrm{~mm}$. These alloys are known as bulk-metallic glasses, or BMGs. ${ }^{2}$ Many BMGs can be produced by $\mathrm{Cu}$-mold casting, providing the possibility for net-shape casting of complex parts. However, melt flow into the mold, melt temperature and mold temperature can influence the glass forming ability (GFA) and properties of the cast part. Although TPF methods, as already discussed, are intriguing for producing complex parts from BMG feedstock, these methods often require specialized equipment and the processing time is limited. For thermally sensitive BMGs, like the Mg-based alloys, ${ }^{10}$ the TPF ability is likely to be very low, and relaxation effects could negatively affect the properties. Thus, machining techniques that do not appreciably alter the atomic structure of the BMG are necessary for wider industrial application of metallic glasses. Traditional machining methods such as turning and drilling have been examined for Zr-based BMGs, and it was found, that

Further author information: Claus.Dold@inspire.ethz.ch, www.inspire.ethz.ch, +41 (44) 6337956

Laser Applications in Microelectronic and Optoelectronic Manufacturing (LAMOM) XIX, edited by Yoshiki Nakata,

Xianfan Xu, Stephan Roth, Beat Neuenschwander, Proc. of SPIE Vol. 8967, 89670V

(C) 2014 SPIE $\cdot$ CCC code: $0277-786 X / 14 / \$ 18 \cdot$ doi: $10.1117 / 12.2035383$

Proc. of SPIE Vol. 896789670 V-1 
Table 1. Laser systems used for the experiments

\begin{tabular}{lll}
\hline laser active medium & pulsewidth $[\mathrm{ps}]$ & wavelength $[\mathrm{nm}]$ \\
\hline Ti:Sapphire & 0.100 & 800 \\
Yb:Glass & 0.410 & 1030 \\
Nd:YAG & 12 & 1064 \\
\hline
\end{tabular}

at high temperatures (about $2700 \mathrm{~K}$ ), high strain and tool damage can occur. ${ }^{11-13}$ Surface structuring of BMGs is also intriguing for microelectromechanical systems (MEMS) applications and biomedical devices. ${ }^{14,15}$ Laser processing is a promising method. However, similar to the machining techniques already mentioned, a "cold" laser method is needed to avoid crystallization. Recently, numerous studies have examined the use of ultra-short (ps and fs) laser pulses in surface structuring of conventional engineering metals, ${ }^{16-18}$ however, similar research for metallic glasses is lacking. Wang et $\mathrm{al}^{19}$ applied this technique to a $\mathrm{Zr}$-based BMG, determining the ablation threshold and studying the morphology of the cut surfaces. That study, however, did not fully address the application of the technique to producing patterned structures. Here, we perform a detailed analysis of the effects of variation of laser parameters on the surface of a well-studied $\mathrm{Zr}-\mathrm{Cu}$-Fe-Al BMG, ${ }^{20}$ which is intended for use in biomedical applications. ${ }^{21}$ Occurrence of re-casting and associated effects on microsctructure for the various conditions will be presented and discussed. Optimal process conditions are determined for structuring with minimal damage to the glass matrix, including single-pulse ablation. An automated routine is developed to apply these optimized conditions in generating a pitted grid of varying depth and pit spacing, which can be used to determine cell response for bone implants made from this biocompatible, Ni-free BMG.

\section{EXPERIMENTAL METHODS}

\subsection{Sample preparation and laser system setup}

Amorphous specimens of $Z r_{62.5}-C u_{22.5}-F e_{5}-A l_{10}$ (at. \%) alloy are prepared by suction casting into a $\mathrm{Cu}$-mold during electrical-arc melting on a water-cooled $\mathrm{Cu}$ hearth. Two mold geometries are used: rectangular plates $4 \times 32 \times 1 \mathrm{~mm}$ and cylinders of $4 \mathrm{~mm}$ diameter and $32 \mathrm{~mm}$ length. Prior to structuring, samples are polished by a sequence of $\mathrm{SiC}$ grinding paper up to 4000 grit and cleaned in an ultrasonic bath in a mixture of acetone and ethanol. The experimental setup consists of three laser systems with different pulsewidths and wavelengths (see table 1). Beam scanning is realized via two axis scanning systems. The beam is guided either through the scanning mirrors which project onto a lens and then through a microscope objective with a $5 \mathrm{x}$ or $10 \mathrm{x}$ magnification and a numerical aperture of $\mathrm{NA}=0.15$ and $\mathrm{NA}=0.3$ respectively. On experiments using a scanhead for beam delivery, plane field correction is achieved using a lens with a focal length of $f=120 \mathrm{~mm}$ in combination with a device which enables shifting the focal spot in Z-direction. The samples are attached to a dual micrometer driven goniometer stage, which itself is mounted on a table which translates in $\mathrm{x}-$, $\mathrm{y}-$ and z-direction with a resolution of $20 \mathrm{~nm}$.

\subsection{Sample characterization}

Characterization of the sample surface after structuring is done via three dimensional optical microscopy, using a 20x microscope objective lens, a vertical resolution of $50 \mathrm{~nm}$ and a lateral resolution of $1 \mu \mathrm{m}$. Measurement lengths range between 100 to $350 \mu \mathrm{m}$. In this way, the absolute $R_{\mathrm{z}}$ and average $R_{\mathrm{a}}$ values are determined. Profilometer measurements are used to determine volume ablation rates by measuring cross-sections of the profile and multiplying these values by a standard length. A scanning electron microscope (SEM) evaluation to analyze surface topographies is performed as well as energy dispersive X-ray spectroscopy (EDX) analysis for determining the composition in and around the structured areas. Crystallization effects are studied using X-ray diffraction (XRD), differential scanning calorimetry (DSC) and EDX measurements. For the DSC studies, BMG cylindrical sections, which are $5 \mathrm{~mm}$ long and $3 \mathrm{~mm}$ in diameter are treated by the different laser conditions. Between each treated section, an equally-sized section is left untreated and used to determine the DSC trace for the as-cast BMG. 


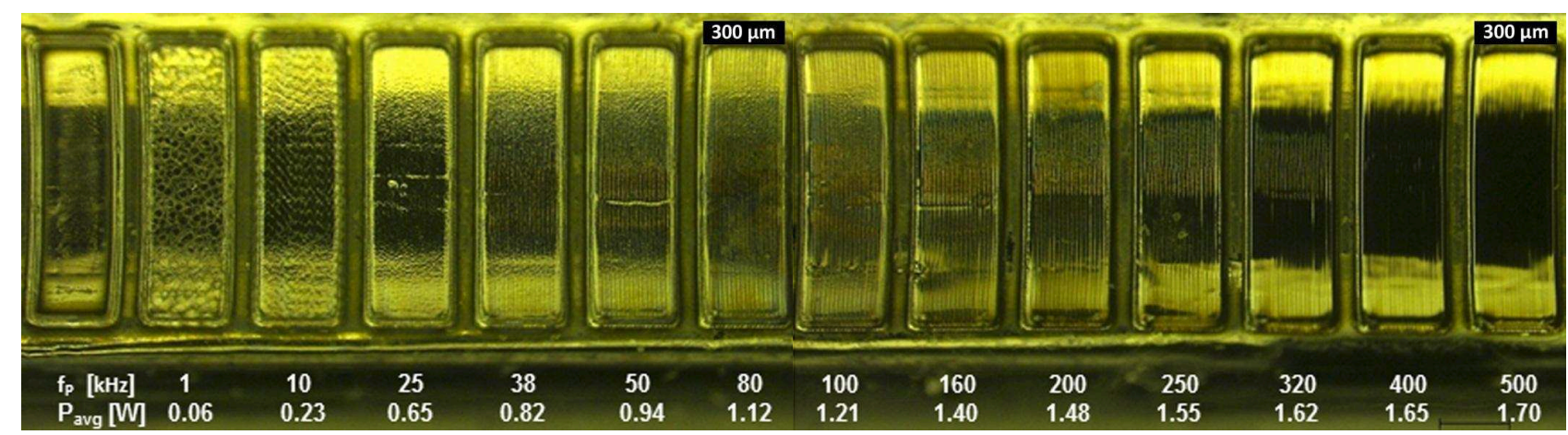

Figure 1. Surface structuring on an unpolished rotation-symmetric rod geometry by repetition rate variation using a laser system with the following parameters $\tau=12 \mathrm{ps}, v_{\mathrm{f}}=400 \mathrm{~mm} / \mathrm{s}, \Delta l=8 \mu \mathrm{m}$, \# $\#_{\mathrm{rep}}=10$. Applied fluence values are (left to right) $F=0.59$ to $13.48 \mathrm{~J} / \mathrm{cm}^{2}$.

\section{RESULTS AND DISCUSSION}

Surface ablation characteristics are controlled by variation of the pulse energy, repetition rate, laser pulse- and scanning line offset. Each parameter is tested in a separate experimental series. The resulting surfaces range from fine pits ( 1 to $2 \mu \mathrm{m}$ diameter and depth) to nearly polished surfaces at repetition rates of a few hundred $\mathrm{kHz}$. An example of such a surface series is shown in figure 1. Repetition rate variation also changes average power and therefore also the pulse energy. With respect to surface quality, the main influencing factors are pulse to pulse overlap as well as pulse energy. For different surface characteristics in these series the scanning velocity is kept constant at $v_{\mathrm{f}}=400 \mathrm{~mm} / \mathrm{s}$ and the repetition rate is varied. Low repetition rates of $f_{\mathrm{P}}=1$ to $100 \mathrm{kHz}$ exhibit a coarse surface, owing to a large pulse separation value ranging from $\Delta x=4$ to $400 \mu \mathrm{m}$ or $\Delta x \cong-1566$ to $66 \%$. A large value $\Delta x=400 \mu \mathrm{m}$ with a focal diameter of $d_{\text {foc }}=10 \mu \mathrm{m}$ represents an interpulse separation distance of $\Delta x=390 \mu \mathrm{m}$, therefore the pulses do not overlap. Since repetition rate values are low, fluence values are the highest within this series, ranging from $F=4.16$ to $13.48 \mathrm{~J} / \mathrm{cm}^{2}$. Volume removal rates are minimal because of the low pulse overlap values. Higher repetition rates and therefore larger pulse overlap values produce a much more even and smoother surface. A fine repetitive line structure can also be seen in the vertical direction since line spacing is around $\Delta l=8 \mu \mathrm{m}$ with a focal spot size of $d_{\mathrm{foc}}=24 \mu \mathrm{m}$. By increasing the applied repetition rate, between $f_{\mathrm{P}}=320$ to $500 \mathrm{kHz}$, a fine line structure or even polished surface can be achieved, even though the initial sample surface quality is as-cast in structure. All other parameters are kept constant within this series. A material composition study is shown in table 2. The experiment is carried out on a separately laser treated area of BMG and the EDX analysis is achieved by using a backscattering electron detector on as-cast and laser irradiated material is applied on an outer region and also within the ablation crater. The sample geometry shows a polished surface and the processed ablation crater is cut and polished to a cross-sectional part. The values in the table represent atomic percentage values. Oxygen and gold elements, which are also detected, are removed from the table for comparison against nominal and as-cast material compositions. The former occurs due to laser processing in ambient air leads to formation of a surface oxide layer, the latter is used for gold sputtering before SEM and EDX analysis. An analysis of pulsewidth influence on the ablated surface structure by applying three different pulsewidths starting at a fluence just above the ablation threshold. SEM images are presented in figure 2 for pulsewidths of $\tau=12 \mathrm{ps}$, in figure 3 for $\tau=410 \mathrm{fs}$, and in figure 4 for $\tau=100 \mathrm{fs}$. In each of

Table 2. EDX analysis, spectrum evaluation (in at. \%) of as-cast and at various measurement points around the processing location. Laser parameters used are $P_{\mathrm{avg}}=25 \mathrm{~mW}, f_{\mathrm{P}}=1 \mathrm{kHz}, e_{\mathrm{P}}=25 \mu \mathrm{J}, F=2.97 \mathrm{~J} / \mathrm{cm}^{2}, d_{\mathrm{foc}}=32.76 \mu \mathrm{m}$

\begin{tabular}{ccccc}
\hline Element & nominal & as-cast & crater side & mid crater \\
\hline $\mathrm{Zr}$ & 62.50 & 62.88 & 33.58 & 64.41 \\
$\mathrm{Cu}$ & 22.50 & 23.53 & 44.52 & 21.49 \\
$\mathrm{Al}$ & 10.00 & 9.37 & 16.18 & 10.28 \\
$\mathrm{Fe}$ & 5.00 & 4.21 & 5.72 & 3.81 \\
\hline
\end{tabular}



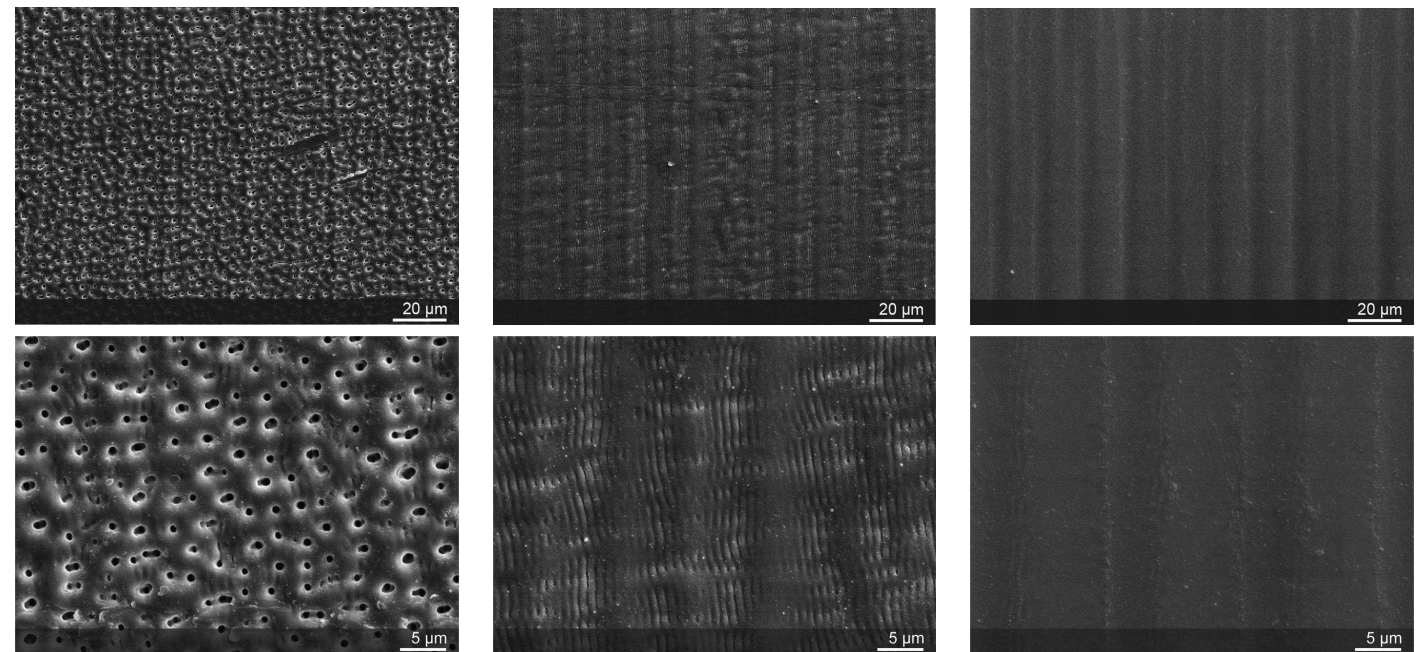

Figure 2. Sample surface after structuring with a pulsewidth of $\tau=12$ ps and increasing fluence $F=0.22$ to $1.33 \mathrm{~J} / \mathrm{cm}^{2}$.
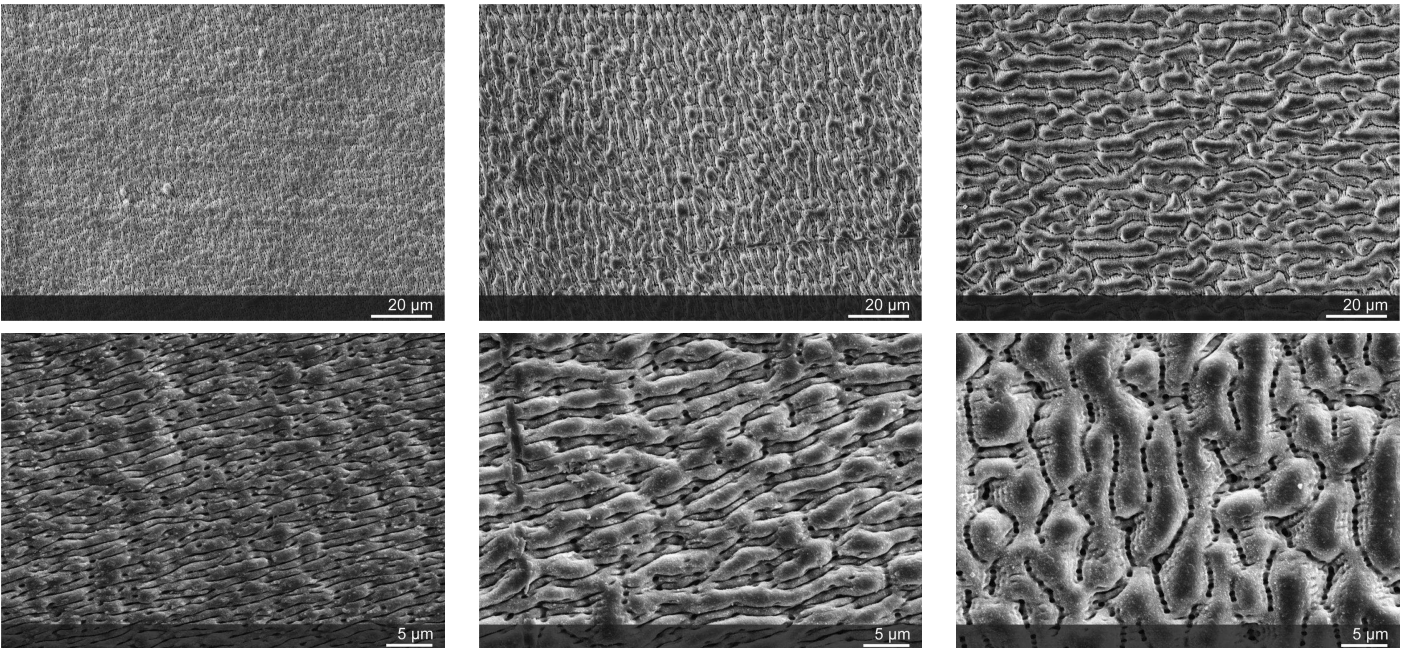

Figure 3. Sample surface after structuring with a pulsewidth of $\tau=410 \mathrm{fs}$ and increasing fluence $F=0.103$ to $0.206 \mathrm{~J} / \mathrm{cm}^{2}$.
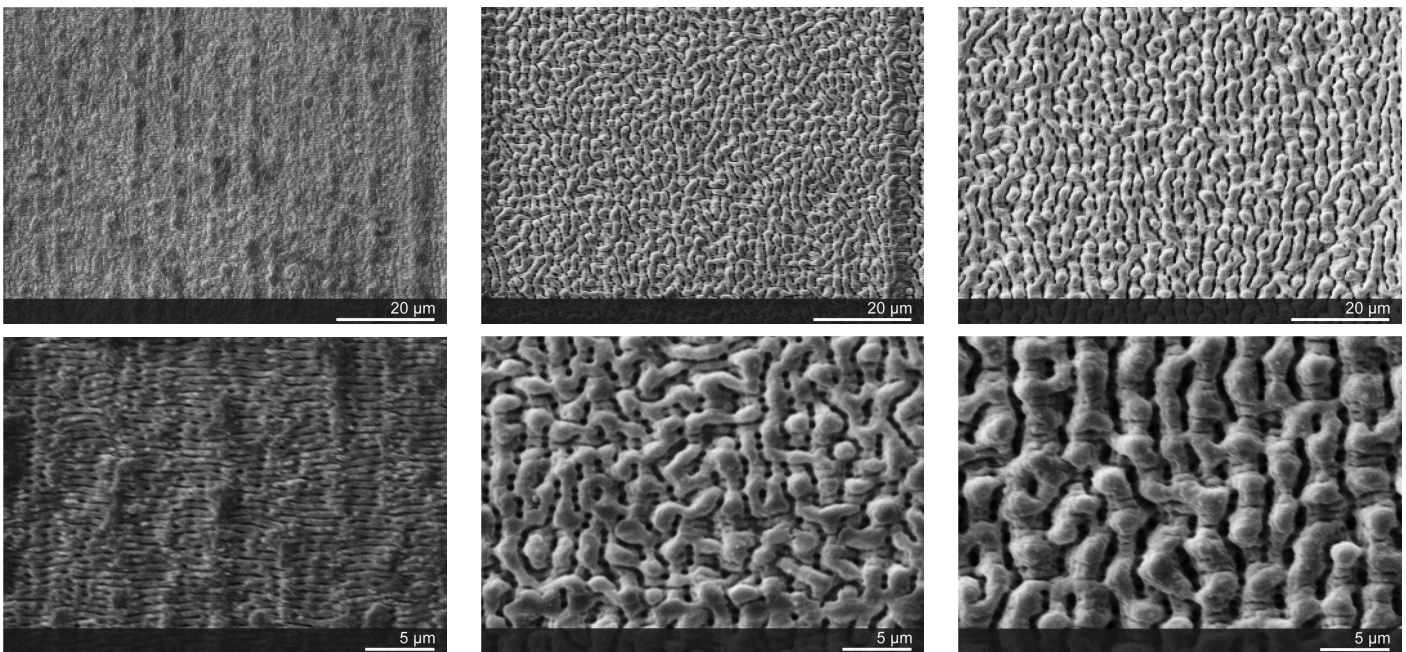

Figure 4. Sample surface after structuring with a pulsewidth of $\tau=100 \mathrm{fs}$ and increasing fluence $F=0.2$ to $1.09 \mathrm{~J} / \mathrm{cm}^{2}$. 


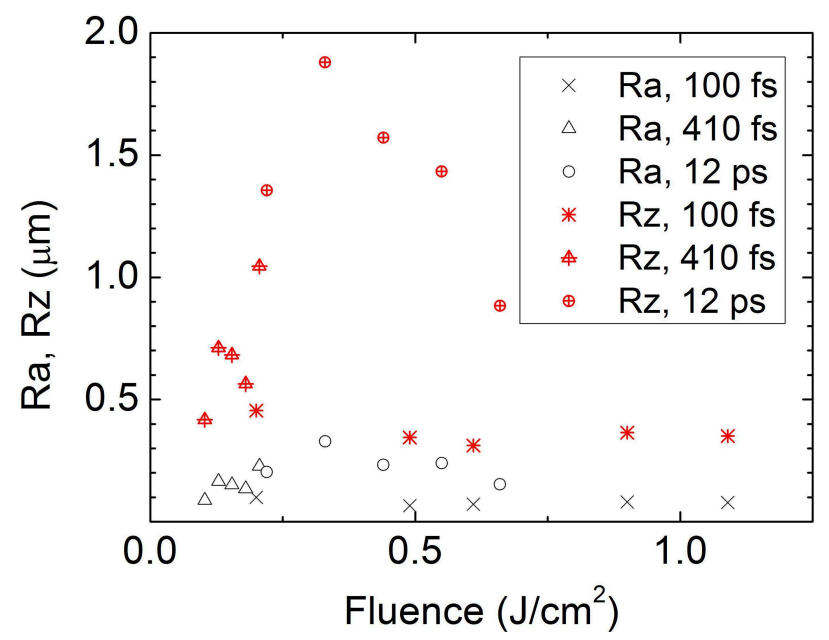

Figure 5. Surface roughness parameters $R_{\mathrm{a}}$ and $R_{\mathrm{z}}$ at different pulsewidths $\tau=100 \mathrm{fs}, 410 \mathrm{fs}, 12 \mathrm{ps}$.

these figures, the surface morphology is evaluated and the bottom row of images shows a higher magnification image of the structured surface shown in the picture directly above. The variable parameter in all three series is the average power, and therefore the pulse energy. In the picosecond ablation series (figure 2) the average power is varied from $P_{\mathrm{avg}}=0.5$ to $3 \mathrm{~W}$ at a processing speed of $v_{\mathrm{f}}=1000 \mathrm{~mm} / \mathrm{s}$ and a repetition rate of $f_{\mathrm{P}}=500 \mathrm{kHz}$. For the $\tau=410 \mathrm{fs}$ series (figure 3), the variation of average power is $P_{\mathrm{avg}}=0.08$ to $0.018 \mathrm{~W}$ at a processing speed of $v_{\mathrm{f}}=300 \mathrm{~mm} / \mathrm{s}$ and a repetition rate of $f_{\mathrm{P}}=1 \mathrm{MHz}$, and for $\tau=100 \mathrm{fs}$ (figure 4) the average power values are $P_{\mathrm{avg}}=170$ to $550 \mu \mathrm{W}$ using $v_{\mathrm{f}}=2 \mathrm{~mm} / \mathrm{s}$ as processing speed with a repetition rate of $f_{\mathrm{P}}=1 \mathrm{kHz}$. Figure 2 shows that picosecond pulses can be used to generate a pitted surface, with average pit diameter and depth of about 1 to $2 \mu \mathrm{m}$, as well as fine ripple structures and nearly polished surface qualities as the pulse energy increases. Femtosecond ablation exhibits a finely rippled surface with features in the submicron range. Composition changes throughout the evaluated areas are minimal. Surface roughness measurements (figure 5), in terms of the averaged roughness value $R_{\mathrm{a}}$ shows a small rise at low fluences, but is then confined within a narrow band of values (at higher fluences). For the experiments using $\tau=12 \mathrm{ps}$ pulsewidth, notably different behavior is observed for $R_{\mathrm{a}}$ and $R_{\mathrm{z}}$ as the fluence increases. These do show a peak at about $R_{\mathrm{a}}=0.3 \mu \mathrm{m}$, which decreases again as fluence increases. For $\tau=100 \mathrm{fs}$, the average surface roughness reaches values between $R_{\mathrm{a}}=0.066$ to $0.1 \mu \mathrm{m}$, although a submicron-sized surface structure can be seen, as shown in figure 4. For a pulsewidth of $\tau=410 \mathrm{fs}$, a quick rise in surface roughness from $R_{\mathrm{a}}=0.088$ to $0.227 \mu \mathrm{m}$ and $R_{\mathrm{z}}=0.417$ to $1.045 \mu \mathrm{m}$ is detected as fluence values rise from $F=0.103$ to $0.206 \mathrm{~J} / \mathrm{cm}^{2}$. For $\tau=12 \mathrm{ps}$ the values range from $R_{\mathrm{a}}=0.204$ to $0.153 \mu \mathrm{m}$ and there is perhaps a weak peak around $R_{\mathrm{a}}=0.329 \mu \mathrm{m}$ at a fluence of $F=0.33 \mathrm{~J} / \mathrm{cm}^{2}$. Femtosecond irradiation leads to a less homogenous surface structure in the submicron range, with nanoripple formation on the surface, which is typical in many processes using a pulsewidth in the femtosecond range. Okamoto et al. describe the ablation effects using femtosecond pulses on metal targets and microstructure formation in the cut groove. Surface patterning is related to the intensity ditribution at the focal point and can be calculated by diffraction theory. ${ }^{22} R_{\mathrm{a}}$ and $R_{z}$ are much lower for femtoseceond compared to picosecond pulses. The waviness values for the surface are not considered but could also have an effect on the roughness parameters. To determine the effects of the laser processing on the amorphous material, DSC and XRD are performed. DSC measurements are made for four samples, an untreated (as-cast), and three laser processed samples with pulsewidths of $\tau=125 \mathrm{~ns}, 12 \mathrm{ps}, 410$ fs. Crystallization effects are expected for pulse durations higher than $\tau=10 \mathrm{ps}$ due to thermal ablation properties. From figure 6 (a), no influence of laser irradiation can be detected; all samples show similar values of the glass transition temperature and the enthalpy of primary crystallization remains unchanged outside of error. This unexpected result could be due to the fact that the irradiated volume-fraction of the sample is too small to be detected using this technique. XRD measurements for the same set of conditions are shown in figure 6 (b), these are also shown in Wortmann et al. ${ }^{23}$ with respect to the discussion of energy transport mechanisms on ultrafast time scales. From that figure it can be seen that crystallization occured on the surface for the ns and ps conditions, indicated by the sharp 

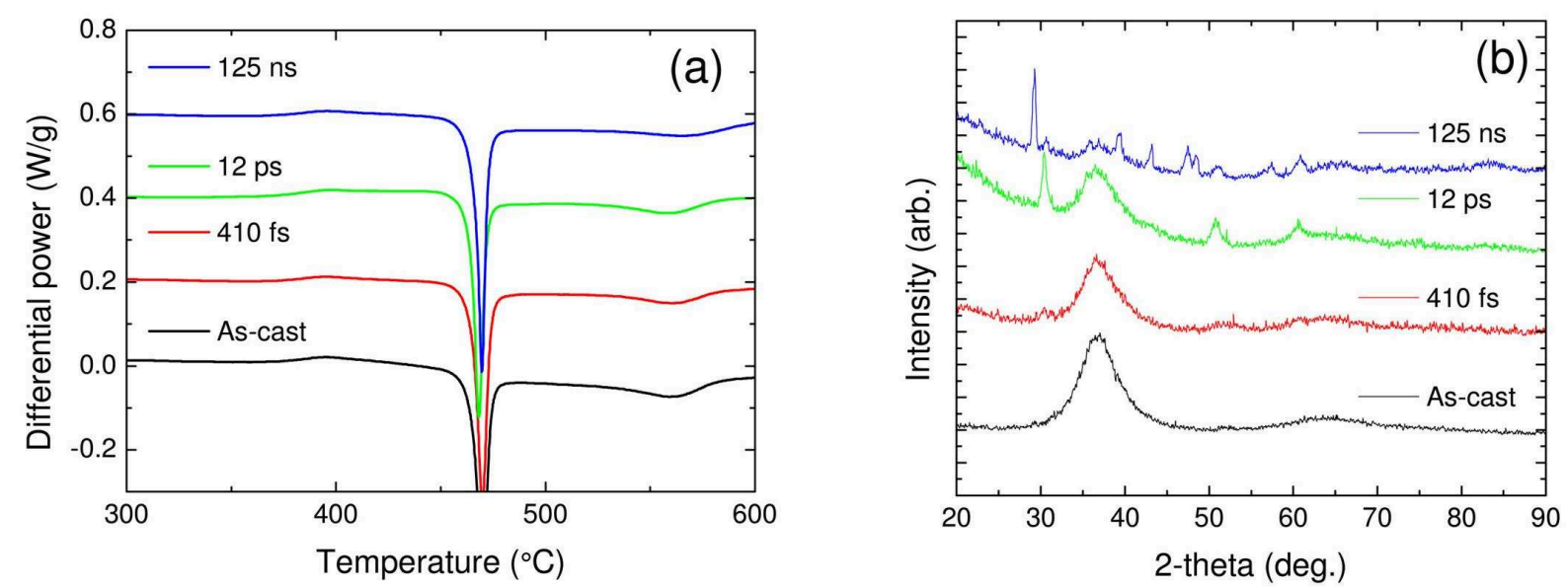

Figure 6. (a)Differential scanning calorimetry and (b) XRD analysis of untreated (as-cast) and irradiated sample using multiple pulsewidths $\tau=410 \mathrm{fs}, 12 \mathrm{ps}, 125 \mathrm{~ns}$.

peaks corresponding to Bragg reflections. In contrast, the as-cast condition and the sample that is treated with a pulsewidth of $\tau=410 \mathrm{fs}$ show a similar amorphous character. From figure 6 (b) it is evident that the phases that crystallize on the surface are different for the ns and ps conditions. One explanation is that for the ns beam, local heating is too strong and recrystallization effects are visible, while the ps beam produces a crystalline oxide layer, as it is known to form during exposure of Zr-based BMGs to air at elevated temperatures which can be seen in figure 7. This oxide region has a width of approximately 0.5 to $1 \mu \mathrm{m}$ and EDX analysis of the oxide layer region shows a significant depletion in zirconium and an enrichment in aluminium. Generation of a pitted surface structure is carried out by translating the workpiece on a three axis piezo driven stage assembly with a linear axis resolution of $50 \mathrm{~nm}$, as described in section 2.1. Ablation threshold determination is first performed for single and multipulse treatment of the sample surface. The ablation threshold is then calculated via an extrapolation of the measured squared diameters against the logarithm of the relevant fluence values of each experimental series. For single-pulse ablation, this results in an ablation threshold of $F_{\mathrm{th}, \mathrm{s}} \cong 300 \mathrm{~mJ} / \mathrm{cm}^{2}$, which is close to the value of Wang et al. ${ }^{19}$ at $F_{\mathrm{th}, \mathrm{s}(\text { Wang })} \cong 210 \mathrm{~mJ} / \mathrm{cm}^{2}$. For multipulse ablation, the found threshold value is $F_{\mathrm{th}, \mathrm{mp}} \cong 88 \mathrm{~mJ} / \mathrm{cm}^{2}$. These values are valid for a pulsewidth range of $\tau=100 \mathrm{fs}$ to 5 ps at a wavelength of $\lambda=800 \mathrm{~nm}$. As mentioned in section 1, surface-structured BMGs are of interest for applications including biomedical devices. For bone implants, the effect of a rough or pitted surface on cell adhesion and

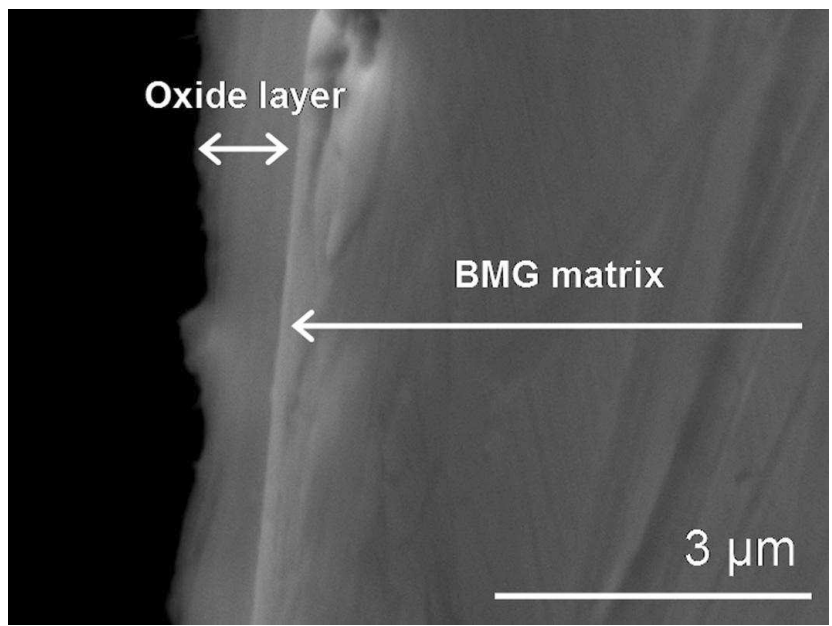

Figure 7. SEM image of cross section of ablated crater region. An Al-enriched surface oxide layer can be detected. Used laser parameters are $\tau=100 \mathrm{fs}, P_{\mathrm{avg}}=25 \mathrm{~mW}, f_{\mathrm{P}}=1 \mathrm{kHz}, F=2.966 \mathrm{~J} / \mathrm{cm}^{2}$. 


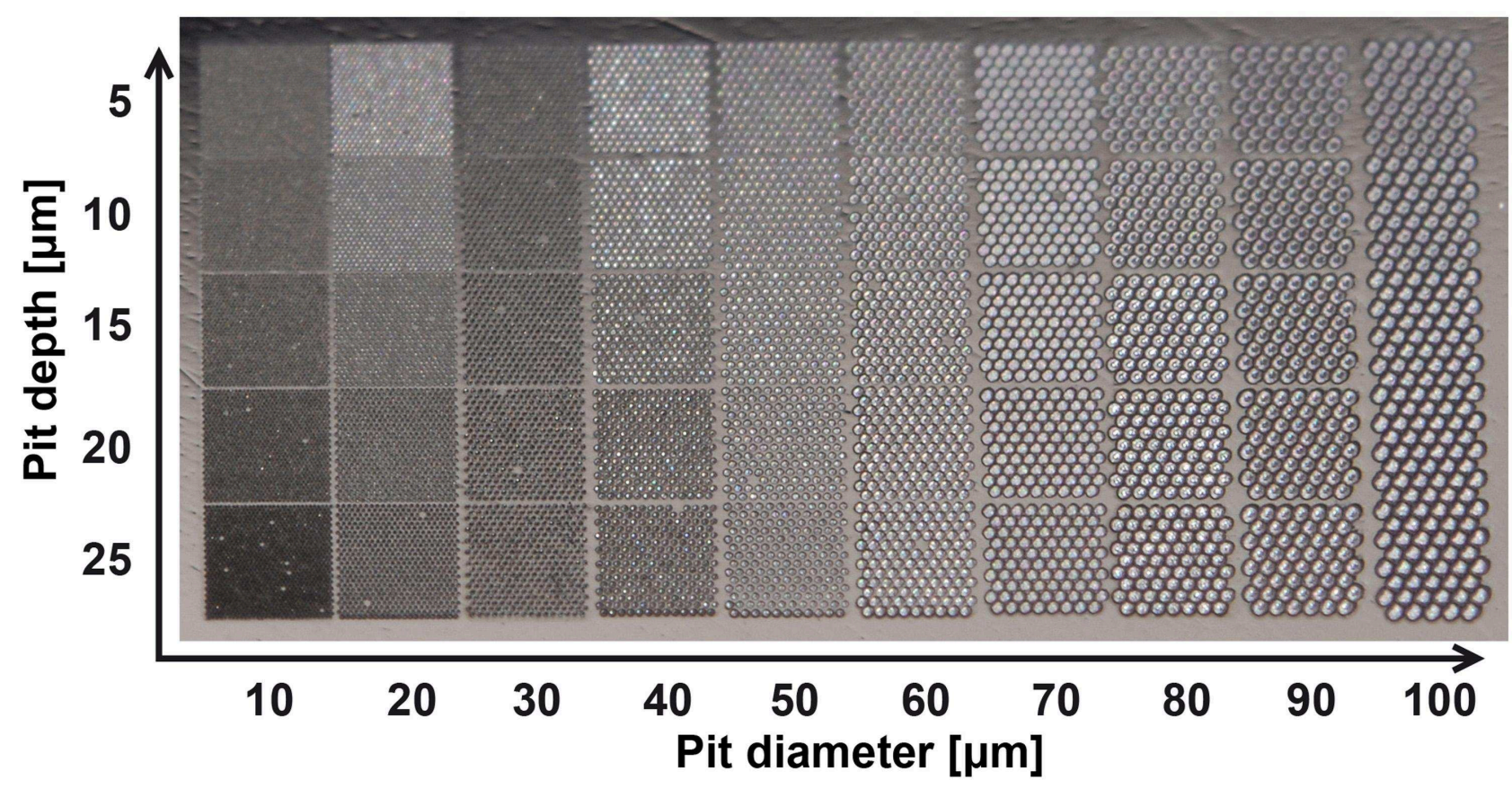

Figure 8. Pitted surface grid with pit diameters ranging from $d=10$ to $100 \mu \mathrm{m}$ and a pit depth of $\Delta z \cong 10$ to $50 \mu \mathrm{m}$ using a pulsewidth of $\tau=100 \mathrm{fs}$ with an individual quadratic field size of $A=0.8 x 0.8 \mathrm{~mm}^{2}$.

division remains a subject of debate. In order to test these effects for the Zr-based BMG studied here, a sample has been structured with a field of pits with varying pit diameters and depths, as shown in figure 8. Different pit diameters are achieved by defocusing the beam and using two microscope objectives. Diameter variation is achieved in the range of $d=10$ to $100 \mu \mathrm{m}$ in $\Delta d=10 \mu \mathrm{m}$ steps. The pits with $d=10$ to $70 \mu \mathrm{m}$ are made using a Zeiss Epiplan-Neofluar microscope objective with 5x magnification and a numerical aperture of $\mathrm{NA}=0.15$. Larger diameters are done using a Zeiss EC Plan-Neofluar objective with 10x magnification and a numerical aperture of $\mathrm{NA}=0.3$. The pits are layed out with a densely-packed structure to achieve a homogenous distribution of treated to untreated surface. Depth variations are achieved by varying the number of pulses per area $n_{\mathrm{p}}=150$ to 650 . Maximum depths attained in this experiment are about $\Delta_{z} \cong 50 \mu \mathrm{m}$. Experiments on evaluating the changes in bone cell response to such a grid are ongoing.

\section{CONCLUSIONS AND OUTLOOK}

Zr-based bulk metallic glasses can be processed without noticable crystallization effects with high surface quality for low fluence values in the range of $0.1<F<1.1 \mathrm{~J} / \mathrm{cm}^{2}$ in the femtosecond pulsewidth regime and with an oxide layer in the range of about one micron thickness in the picosecond pulsewidth regime. A multitude of surface properties can be achieved using different pulsewidths from a nearly polished surface to coarse grainlike structures with $0.06<R_{\mathrm{a}}<0.3 \mu \mathrm{m}$. Surface ripple structures can be controlled by using different pulsewidth regimes in terms of laser radiation. Pitted surface structures can be manufactured with pit diameters in the range of $d=10$ to $100 \mu \mathrm{m}$ and a depth range of $\Delta z \cong 10$ to $50 \mu \mathrm{m}$. One possible application for such pitted surface structures is in biomedical implants, particularly for osteosynthesis, due to the biocompatibility of the $\mathrm{Zr}-\mathrm{Cu}-\mathrm{Fe}-\mathrm{Al} \mathrm{BMG}$ and it's good mechanical properties. Further work in this direction is ongoing.

\section{ACKNOWLEDGMENTS}

This joint work originated in an IDEA League exchange between ETH Zurich and the chair for laser technology at the RWTH in Aachen. Therefore we would like to thank the IDEA League project for expense covering. 


\section{REFERENCES}

[1] A. Inoue, "Stabilization of metallic supercooled liquid and bulk amorphous alloys," Acta materialia 48, pp. 279-306, 2000.

[2] J.-F. Loeffler, "Bulk metallic glasses," Intermetallics 11, pp. 529-540, 2003.

[3] W. H. Wang, C. Dong, and C. H. Shek, "Bulk metallic glasses," Materials science and engineering 44, pp. 529-540, 2003.

[4] M. F. Ashby and A. L. Greer, "Metallic glasses as structural materials," Scripta Materialia 54, pp. 321-326, 2006.

[5] R. Busch, J. Schroers, and W. H. Wang, "Thermodynamics and kinetics of bulk metallic glass," MRS Bulletin 32(8), pp. 620-623, 2007.

[6] J. Schroers, "Processing of bulk metallic glass," Advanced Materials 22(8), pp. 1566-1597, 2010.

[7] J. Schroers and N. Paton, "Amorphous metal alloys," Advanced Materials 85 Processes 164(8), pp. 61-63, 2006.

[8] W. L. Johnson, G. Kaltenboeck, M. D. Demetriou, J. P. Schramm, X. Liu, K. Samwer, C. P. Kim, and D. C. Hofmann, "Beating crystallization in glass-forming metals by millisecond heating and processing," Science 332(6031), pp. 828-833, 2011.

[9] W. Klement, R. H. Willens, and P. O. Duvez, "Non-crystalline structure in solidified gold-silicon alloys," Nature 187, pp. 869-870, 1960.

[10] X. Gu, G. J. Shiflet, F. Q. Guo, and S. J. Poon, "Mg-Ca-Zn bulk metallic glasses with high strength and significant ductility," J. Mater. Res. 20(8), pp. 1935-1938, 2005.

[11] M. Bakkal, C. T. Liu, R. Watkins, R. O. Scattergood, and A. H. Shih, "Oxidation and crystallization of Zr-based bulk metallic glass due to machining," Intermetallics 12(2), pp. 195-204, 2004.

[12] M. Bakkal, A. J. Shih, R. O. Scattergood, and C. T. Liu, "Machining of a Zr-Ti-Al-Cu-Ni metallic glass," Scripta Materialia 50(5), pp. 583-588, 2004.

[13] M. Bakkal, A. J. Shih, S. B. McSpadden, and R. O. Scattergood, "Thrust force, torque, and tool wear in drilling the bulk metallic glass," International Journal of Machine Tools \& Manufacture 45(7-8), pp. 863$872,2005$.

[14] B. Zberg, P. J. Uggowitzer, and J.-F. Loeffler, "Mg-Zn-Ca glasses without clinically observable hydrogen evolution for biodegradable implants," Nature Materials 8, pp. 887-891, 2009.

[15] V. Wessels, G. L. Mene, S. Fischerauer, T. Kraus, A. M. Weinberg, P. J. Uggowitzer, and J.-F. Loeffler, "In vivo performance and structural relaxation of biodegradable bone implants made from Mg--Zn-Ca bulk metallic glasses," Advanced Engineering Materials 14(6), pp. B357-B364, 2012.

[16] M. Lenzner, J. Krueger, S. Sartania, Z. Cheng, C. Spielmann, G. Mourou, W. Kautek, and F. Krausz, "Femtosecond optical breakdown in dielectrics," Phys. Rev. Lett. 80(18), pp. 4076-4079, 1998.

[17] H. Dachraoui, W. Husinsky, and G. Betz, "Ultra-short laser ablation of metals and semiconductors: evidence of ultra-fast coulomb explosion," Applied Physics A 83(2), pp. 333-336, 2006.

[18] P. T. Mannion, J. Magee, E. Coyne, G. O'Connor, and T. Glynn, "The effect of damage accumulation behaviour on ablation thresholds and damage morphology in ultrafast laser micro-machining of common metals in air," Applied Surface Science 233(1-4), pp. 275-287, 2004.

[19] X. Wang, P. Lu, N. Dai, Y. Li, C. Liao, Y. Chang, G. Yang, and Q. Zeng, "Morphology and oxidation of Zr-based amorphous alloy ablated by femtosecond laser pulses," Applied Physics A 89, pp. 547-552, 2007.

[20] K. Jin and J.-F. Loeffler, "Bulk metallic glass formation in $\mathrm{Zr}-\mathrm{Cu}-\mathrm{Fe}-\mathrm{Al}$ alloys," Applied Physics Letters 86(24), pp. 241909 - 241909-3, 2005.

[21] S. Buzzi, K. Jin, P. Uggowitzer, S. Tosatti, I. Gerber, and J.-F. Loeffler, "Cytotoxicity of Zr-based bulk metallic glasses," Intermetallics 14(7), pp. 729-734, 2006.

[22] T. Okamoto, E. Ohmura, T. Sano, Y. Morishige, and I. Miyamoto, "Analytical study on metal microstructures using femtosecond laser," Applied Physics A 81(3), pp. 639-643, 2005.

[23] D. Wortmann, M. Reininghaus, J. Finger, C. Dold, P. Russbueldt, and R. Poprawe, "The physics in applications of ultrafast lasers," Proceedings of SPIE 8306, pp. 830603-830603-6, 2011. 